\title{
Morphological and Molecular Characterization and Relationships of Turkish Local Eggplant Heirlooms
}

\author{
Hatice Filiz BOYACI*, Volkan TOPCU, Akin TEPE, Isilay Karasahin YILDIRIM, \\ Mehmet OTEN, Aytekin AKTAS
}

Bati Akdeniz Agricultural Res. Inst., Dept. of Vegetable Crops and Ornamentals, 07100 Antalya,

Turkey;filiz_boyaci@yahoo.com ("correspondingauthor)

\begin{abstract}
A total of 38 eggplant genotypes, of which 32 were heirloom accessions collected from different regions of Burdur province five were different local genotypes from other provinces, and one was a cultivar, were used as reference in this study. The phylogenetic relationships among these heirlooms were evaluated using 40 morphologic descriptors and five randomly amplified polymorphic RAPD markers. The horizontal dendrograms were created by using UPGMA with both morphologic and molecular data. Burdur heirloom accessions showed high genetic diversity based on morphological and molecular data. The genetic similarity rates ranged from 0.29 to 0.91 according to the morphological data, and ranged from 0.84 to 0.98 according to the molecular data. Molecular data generated by RAPD method, compared to morphological data, were insufficient to reveal genetic diversity. Therefore, in order to confirm genetic variations, studies based on other molecular methods are necessary. The regional genetic populations include a wide eggplant genetic diversity which can be good source for the breeding studies performed in the future.
\end{abstract}

Keywords: accession, breeding, diversity, genetic variation, phylogenetic relationship, RAPD, UPGMA

\section{Introduction}

Eggplant, which was called as the king of vegetables (Daunay and Janick, 2007), is an indigenous plant of India (Weese and Bohs, 2010). It has been well known since BC III and cultivated for 1,500 years in Asia (Kashyap et al., 2003). It is cultivated as a perennial in tropical areas, while it is cultivated as annual in subtropical areas (Kowalska, 2008). Eggplant is placed in Solanum genus and includes wide genotypic and phenotypic variation (Fukuoka et al., 2010). Eggplant is thought to have been developed from the wild ancestor, Solanum insanum has small, round, green, thick-skinned and bitter taste fruits (Barchi et al., 2010). Cultivation $S$. insanum of had been performed in China, India and Thailand (Daunay et al., 2001). Large fruiting eggplants were cultured in India in early time, and small fruiting was cultured at IV century in China and at IX century in Africa (Sekera et al., 2007). First cultivated eggplants were described as high tall plants, with big spines on the calyx, small, bitter fruiting and with high seed content in fruits (Swarup, 1995). Mutation, natural pollination and hybridization, together with selection gave rise to genetic diversity, as well as in decreasing of prickles and bitterness at fruit, changing of fruit shape, size and color (Frary et al., 2007). Genetic diversity accumulated and many different heirlooms emerged in countries where it was cultivated (Prohens et al., 2003). Entrance of the eggplant to Turkey was carried out by the silk-road. The genetic diversity accumulated in producing areas and by trade of eggplant throughout centuries in Anatolia (Janick, 2001). Eggplant cultivation was done in open field until the second half of the 1970s in Turkey and then cultivation under protected cultivation started. The eggplant cultivation in greenhouse was begun with local varieties. However, the growers preferred hybrid F1 varieties which have the cylindrical and dark purple or black colored fruiting and using of them has become commonly in a short period (Ekiz and Boyaci, 2001). Also, the increase of use F1 hybrids in the open field cultivation was observed in recent years. Steadily decrease was seen in the cultivation of local varieties compared to the hybrids (Cericola et al., 2013). The genetic diversity is low among the genotypes which have dark purple-black fruits (MuñozFalcón et al., 2009). In recent years, one of the important problems faced by eggplant breeding programs, as well as in other species, is a narrowing of the genetic base. To create variations, time-consuming and expensive methods are needed, including mutation breeding, interspecific hybridization and biotechnological approaches. The genetic variation contained by heirlooms among is seen in the previous studies (Demir et al., 2010; Muñoz-Falcón et al., 2008, 2009; Prohens et al., 2003, 2008, 2011). Therefore, collection and characterization of genetic resources is required for the improvement of new varieties. In this respect, 
molecular characterization is more reliable then morphological characterization (Li et al., 2010).

Here we investigate the genetic relationships between Turkish heirlooms using morphological and molecular data is presented here.

\section{Materials and methods}

Morphological and molecular studies were conducted at Bati Akdeniz Agricultural Research Institute, Antalya, Turkey in 2010-2013.

In total 38 materials were used in the study of these. 32 were local heirlooms collected from Burdur province during survey studies. Materials origin, providing method and place/company of origin are given in Table 1 . Long purple commercial variety (YRL 68) and heirlooms originating in other province (YRL 1, YRL 3, YRL 6, YRL 57, and YRL 79) were used as reference cultivars for classification of Burdur province heirlooms.

Five RAPD primers OPH-02, OPL-04, OPB-07, OPO10 , OPL-16 $(10 \mathrm{mer})$ reported as highly polymorphic in previously studies (Demir et al., 2010; Nunome et al., 2001) were selected to detect polymorphisms and identify genetic relationship of the heirlooms.

\section{Survey}

The survey studies were performed at nine different locations in Burdur province in September both in 2010 and 2011. In total 32 materials were collected from different farms. The locations of collected eggplants in Burdur province were represented on the map (Fig. 1) and their geographic coordinates were defined (Table 2).

\section{Morphological observations}

For each accession, the seeds were sown in seedling treys containing peat moss. Seedlings in 4-5 leaves stages were planted in glasshouse. Twenty plants were planted for each genotype. Morphological observations were performed according to 40 descriptors chosen among the International Board for Plant Genetic Resources Institute (IBPGRI), International Union for the Protection of New Varieties of

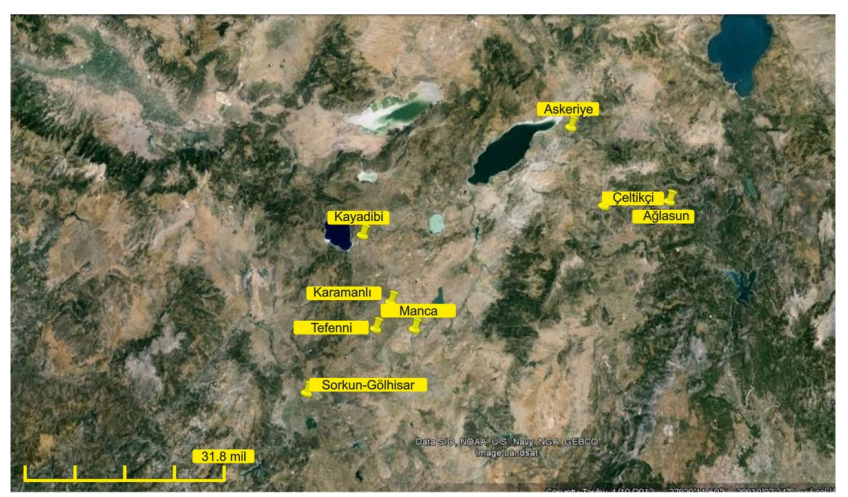

Fig. 1. Geographic map of district and village in Burdur province of Turkey including collection places

Table 1. Origin, providing method and place/company of origin of the materials used in the study

\begin{tabular}{|c|c|c|c|c|}
\hline No & Name of the materials & Origin & Providing method & Place/company of origin \\
\hline 1 & YRL 1 & Antalya-Kumluca District & Agricultural district offices & Grower \\
\hline 2 & YRL 3 & Antalya-Kumluca District & Agricultural district offices & Grower \\
\hline 3 & YRL 6 & Antalya-Kumluca District & Agricultural district offices & Grower \\
\hline 4 & YRL 7 & Burdur-Aglasun /Cine Village & Survey in the field & Grower \\
\hline 5 & YRL 8 & Burdur-Aglasun /Cine Village & Survey in the field & Grower \\
\hline 6 & YRL 9 & Burdur-Aglasun /Cine Village & Survey in the field & Grower \\
\hline 7 & YRL 12 & Burdur/Askeriye Village & Survey in the field & Grower \\
\hline 8 & YRL 14 & Burdur-Aglasun /Cine Village & Survey in the field & Grower \\
\hline 9 & YRL 15 & Burdur-Aglasun /Cine Village & Survey in the field & Grower \\
\hline 10 & YRL 19 & Burdur-Aglasun /Cine Village & Survey in the field & Grower \\
\hline 11 & YRL 20 & Burdur-Aglasun /Cine Village & Survey in the field & Grower \\
\hline 12 & YRL 24 & Burdur-Celtikci/Tekke Village & Survey in the field & Grower \\
\hline 13 & YRL 26 & Burdur-Celtikci/Tekke Village & Survey in the field & Grower \\
\hline 14 & YRL 27 & Burdur-Celtikci/Tekke Village & Survey in the field & Grower \\
\hline 15 & YRL 28 & Burdur-Celtikci/Tekke Village & Survey in the field & Grower \\
\hline 16 & YRL 30 & Burdur-Celtikci/Tekke Village & Survey in the field & Grower \\
\hline 17 & YRL 34 & Burdur-Celtikci/Tekke Village & Survey in the field & Grower \\
\hline 18 & YRL 35 & Burdur-Celtikci/Tekke Village & Survey in the field & Grower \\
\hline 19 & YRL 36 & Burdur-Celtikci/Tekke Village & Survey in the field & Grower \\
\hline 20 & YRL 43 & Burdur-Celtikci/Tekke Village & Survey in the field & Grower \\
\hline 21 & YRL 44 & Burdur-Celtikci/Tekke Village & Survey in the field & Grower \\
\hline 22 & YRL 45 & Burdur-Celtikci/Tekke Village & Survey in the field & Grower \\
\hline 23 & YRL 46 & Burdur-Askeriye Village & Survey in the field & Grower \\
\hline 24 & YRL 47 & Burdur-Askeriye Village & Survey in the field & Grower \\
\hline 25 & YRL 49 & Burdur-Askeriye Village & Survey in the field & Grower \\
\hline 26 & YRL 50 & Burdur-Askeriye Village & Survey in the field & Grower \\
\hline 27 & YRL 51 & Burdur-Askeriye Village & Survey in the field & Grower \\
\hline 28 & YRL 52 & Burdur-Askeriye Village & Survey in the field & Grower \\
\hline 29 & YRL 57 & Mugla-Fethiye/Günesli Village & Survey in the field & Grower \\
\hline 30 & YRL 58 & Burdur-Karamanl1/Manca Village & Survey in the field & Grower \\
\hline 31 & YRL 59 & Burdur-Karamanl1/Manca Village & Survey in the field & Grower \\
\hline 32 & YRL 61 & Burdur-Gölhisar/Sorkum Village & Survey in the field & Grower \\
\hline 33 & YRL 62 & Burdur-Yesilova/Kayadibi Village & Survey in the field & Grower \\
\hline 34 & YRL 64 & Burdur-Tefenni District & Survey in the field & Grower \\
\hline 35 & YRL 65 & Burdur-Tefenni District & Survey in the field & Grower \\
\hline 36 & YRL 68 & Long purple & Purchased & Company \\
\hline 37 & YRL 75 & Burdur City Centrum & Agricultural district offices & Grower \\
\hline 38 & YRL 79 & Antalya-Akseki/Uzumdere Village & Survey in the field & Grower \\
\hline
\end{tabular}


102

Table 2. Geographical coordinates of local heirloom eggplant collected in Burdur province of Turkey

\begin{tabular}{ll}
\hline \multicolumn{1}{c}{ Location } & \multicolumn{1}{c|}{ Geographical coordinates } \\
\hline Aglasun /Cine Village & $37^{\circ} 35^{\prime} 0.07^{\prime \prime N} \mathrm{~N} 30^{\circ} 38^{\prime} 1.34^{\prime \prime} \mathrm{E}$ \\
\hline Celtikci Tekke Village & $37^{\circ} 34^{\prime} 36.86^{\prime \prime} \mathrm{N} / 30^{\circ} 26^{\prime} 40.11^{\prime \prime} \mathrm{E}$ \\
\hline Burdur/Askeriye Village & $37^{\circ} 45^{\prime} 41.88^{\prime \prime} \mathrm{N} / 30^{\circ} 21^{\prime} 15.99^{\prime \prime} \mathrm{E}$ \\
\hline Karamanl1-Manca Village & $37^{\circ} 18^{\prime} 18.74^{\prime \prime} \mathrm{N} / 29^{\circ} 53^{\prime} 19.89^{\prime \prime} \mathrm{E}$ \\
\hline Karamanl-Centrum & $37^{\circ} 22^{\prime} 2.35^{\prime \prime} \mathrm{N} / 29^{\circ} 49^{\prime} 19.71^{\prime \prime} \mathrm{E}$ \\
\hline Gölhisar-Sorkum Village & $37^{\circ} 9^{\prime} 54.49^{\prime \prime} \mathrm{N} / 29^{\circ} 34^{\prime} 39.38^{\prime \prime} \mathrm{E}$ \\
\hline Yesilova-Kayadibi Village & $37^{\circ} 31^{\prime} 19.56^{\prime \prime} \mathrm{N} / 29^{\circ} 44^{\prime} 35.03^{\prime \prime} \mathrm{E}$ \\
\hline Tefenni-Centrum & $37^{\circ} 18^{\prime} 27.39^{\prime \prime} \mathrm{N} / 29^{\circ} 46^{\prime} 40.57^{\prime \prime} \mathrm{E}$ \\
\hline
\end{tabular}

Plants (UPOV) plant feature criteria and some of the criteria for the breeders. Descriptors include plant, leaf, flower and fruit traits observations and measurements (Table 3). Skin color of the each eggplant specimens was measured with a portable tristimulus reflectance colorimeter Minolta CR-400 Chroma Meter (Konica Minolta Sensing, Inc., Osaka, Japan), and the parameters were expressed in CIE $\mathrm{L}^{*} \mathrm{a}^{*} \mathrm{~b}^{*}$ system, where $\mathrm{L}^{*}$ is lightness (brightness-darkness) ranged from 0 to 100 units, $\mathrm{a}^{*}$ is light intensity in red $(+)$ or green $(-)$ spectrum, $\mathrm{b}^{*}$ is intensity in yellow $(+)$ or blue $(-)$ spectrum. Chroma $\left(C: \sqrt{ }\left(\mathrm{a}^{2}+\mathrm{b}^{2}\right)\right)$ measures color saturation or intensity and the hue angle $\left(b^{\circ}=\tan ^{-1}\left(\mathrm{~b}^{*} / \mathrm{a}^{*}\right)\right.$ determines the red, yellow, green, blue, purple, or intermediate colors between adjacent pairs of these basic colors. The $\mathrm{L}^{*}, \mathrm{a}^{*}$, and $\mathrm{b}^{*}, C$ and $h$ ${ }^{\circ}$ values obtained from six samples of each eggplant accession. Every record represents the average of three readings which were taken from the equatorial region, spaced equidistantly.

\section{$D N A$ extractions and PCR analysis}

DNA extractions from young leaves were performed according to a modified Doyle and Doyle (1990) method by using CTAB protocol (Mutlu et al., 2008). RAPD analysis was performed according to Demir et al. (2010). The amplification reactions were released containing 20 ng DNA, 0.5 unite Taq polymerase, $2.5 \mu \mathrm{L} 10 \mathrm{X}$ buffer, $3.5 \mu \mathrm{L} 25 \mathrm{mM} \mathrm{MgCl}_{2}, 2 \mu \mathrm{L} 2.5$ $\mathrm{mM}$ dNTPs, $2 \mu \mathrm{L}$ RAPD primer for RAPD-PCR DNA was amplified in a thermal cycler. It was programmed for an initial 5 min denaturation step at $94^{\circ} \mathrm{C}$, followed by 35 cycles of a $30 \mathrm{~s}$ denaturation step at $94{ }^{\circ} \mathrm{C}, 1 \mathrm{~min}$ annealing at $35^{\circ} \mathrm{C}, 45 \mathrm{~s}$ extension step at $72^{\circ} \mathrm{C}$, followed by a final 8 min extension step at $72^{\circ} \mathrm{C}$.

\section{Statistical analysis}

Genetic similarity was analyzed by the UPGMA (Unweighted pair-group method, arithmetic average) clustering procedure using the software NTSYS (Numerical Taxonomy Multivariate

Table 3. Descriptors used for characterization and evaluation of eggplant accessions used in the study

\begin{tabular}{|c|c|}
\hline Traits & Description \\
\hline Planthabit & Score range ( $1=$ Open, $3=$ Bushy, $5=$ Semi open $)$ \\
\hline Plantheight & Score range ( $1=$ Long, $3=$ Intermediate, $5=$ Short $)$ \\
\hline Stem thickness & Score range ( $1=$ Thick, $3=$ Intermediate, $5=$ Thin $)$ \\
\hline Stem hairiness & Score range $(1=$ Dense, $3=$ Intermediate, $5=$ Tenuous $)$ \\
\hline Stem color & Score range ( $1=$ Grayish, $3=$ Green, $5=$ Green-purple, $7=$ Grayish-green-purple, $9=$ Grayish-green, $11=$ Grayish-purple, $13=$ Purple) \\
\hline Shoot tip color & Score range ( $1=$ Grayish, $3=$ Green, 5=Green-purple, $7=$ Grayish-green-purple, 9=Grayish-green, 11=Grayish-purple, 13=Purple) \\
\hline Length of internodes & Score range ( $1=$ Long, $3=$ =Intermediate, $5=$ Short $)$ \\
\hline Leafcolor & Score range $(1=$ Light green, $3=$ Green, $5=$ Dark green $)$ \\
\hline Leafsize & Score range $(1=$ Large, $3=$ Intermediate, $5=$ Small $)$ \\
\hline Leafhairiness & Score range ( $1=$ Dense, $3=$ Intermediate, $5=$ Tenuous $)$ \\
\hline Presence of spine on petiole & Score range $(1=$ Many, $3=$ Intermediate, $5=$ Few, $7=$ Absent $)$ \\
\hline Budsize & Score range ( $1=$ Large, $3=$ Intermediate, $5=$ Small) \\
\hline Budhairiness & Score range ( $1=$ Dense, $3=$ Intermediate, $5=$ Tenuous, $7=$ Absent $)$ \\
\hline Presence of spine on bud & Score range (1=Many, $3=$ Intermediate, $5=\mathrm{Few}, 7=\mathrm{Absent})$ \\
\hline Flower color & Score range ( $1=$ Light purple, $3=$ Purple, $5=$ Dark purple) \\
\hline Flower size & Score range $(1=$ Large, $3=$ Intermediate, $5=$ Small $)$ \\
\hline Calyx size & Score range $(1=$ Large, $3=$ Intermediate, $5=$ Small $)$ \\
\hline Fruitshape & Score range $(1=$ Long, $3=$ Intermediate, $5=$ Short, $7=$ Ovoid, $9=$ Pear shaped $)$ \\
\hline Dominated fruit color & Score range $(1=$ White, $3=$ Green, $5=$ Purple, $7=$ Black $)$ \\
\hline Range of dominated fruit color & Score range $(1=$ Regular, $3=$ Mottled, $5=$ Stripe, $7=$ Mealy $)$ \\
\hline Fruit stalk length & Score range ( $1=$ Long, $3=$ Intermediate, $5=$ Short $)$ \\
\hline Presence of spine on fruit stalk & Score range $(1=$ Few, $3=$ Intermediate, $5=$ Many, $7=$ Absent $)$ \\
\hline Fruit calyx prickles & Score range $(1=\mathrm{Few}(1-5$ pricks $), 3=\mathrm{Mid}(6-20$ pricks $), 5=\mathrm{Many}($ more than 20$)$ \\
\hline Fruit brightness & Score range ( $1=$ Bright, $3=$ Matt $)$ \\
\hline Fruitend shape & Score range $(1=$ Flat, $3=$ Pointed, $5=$ Round $)$ \\
\hline Fruit curvature & Score range $(1=$ Present, $3=$ Absent $)$ \\
\hline Fruit end button size & Score range $(1=$ Large, $3=$ Intermediate, $5=$ Small $)$ \\
\hline Fruit length $(\mathrm{cm})$ & The average measurement of ten fruits \\
\hline Fruit diameter $(\mathrm{cm})$ & The average measurement of ten fruits \\
\hline Average fruit weight (g) & The average measurement of ten fruits \\
\hline Presence of groove on fruit & Score range $(1=$ Present, $3=$ Absent $)$ \\
\hline Fruit flesh firmness & Score range ( $1=$ Tightly, $3=$ Floppy, $5=$ Spongy $)$ \\
\hline Fruit flesh color & Score range $(1=$ Greenish, $3=$ Greenish-cream, $5=$ White, $7=$ White-cream, $9=$ Greenish-white, $11=$ Cream $)$ \\
\hline Presence of hole in fruit & Score range $(1=$ Present, $3=$ Absent $)$ \\
\hline Degree of fruit curvature & Score range $(1=$ Slight, $3=$ Mid, $5=$ Much $)$ \\
\hline Soluble solids & The average measurement of five fruits juice samples \\
\hline The length of fruit coated by calyx & Score range ( $1=$ Less than $20 \%, 3=$ between $20-70 \%, 5=$ More than $70 \%$ ) \\
\hline Tendency to parthenocarpy & Score range ( $1=$ Present, $3=$ Absent $)$ \\
\hline Presence of seed in fruit & Score range $(1=$ Few, $3=$ Intermediate, $5=$ Many $)$ \\
\hline Seed maturity & Score range ( $1=$ Immature, $3=$ Mature) \\
\hline
\end{tabular}


Analysis System) pc 2.2 version (Rohlf, 1998), using morphological and molecular data. For morphological data, each genotype was characterized with description number (Table 3). For molecular analysis data, each genotype was identified for each primer based on the presence (1) and absence (0) of bands. In order to show the variations STANDARDIZATION module was used. Correlation matrix adapted to SIMINT module was used to determine of correlation coefficient. The dendrograms were drawn using the clustering method UPGMA via the SHAN module. The cophenetic correlation coefficient was calculated with Mantel method to evaluate the efficiency of clustering.

\section{Results}

A high morphological diversity was observed among eggplant genotypes characterized by quantitative and qualitative descriptors. Some of the phenotypic observation data related with important variable traits (calyx prickles, fruit size, weight, shape and curvature, skin color, groove etc.) are reported in Table 4. The fruits calyx of all genotypes had prickles. However, nearly $50 \%$ of them had lower numbers of prickles. Most of the genotypes had between $20-70 \%$ of their fruit length covered by the calyx. The degree of fruit curvature in $50 \%$ of the genotypes was slight. Nearly $30 \%$ percent of the genotypes had fruits with grooved appearance. Dominant fruit color was mostly purple and distribution of this color was 50\% regular, 22\% mealy, $18 \%$ mottled, and $2 \%$ striped according to the genotypes. Fruit flesh color was generally white and hole in the fruit was mostly absent. The lowest total soluble solid content (brix value) was 2.8. The highest brix value (6.1) was determined in YRL 15 which was collected from Cine village The average diameter of the fruits ranged from 32.67 to $73.22 \mathrm{~mm}$. Fruit length and fruit weight average ranged between 10.9 and $23.3 \mathrm{~cm}, 0.100$ and $0.235 \mathrm{~kg}$, respectively.

Skin color characteristics of the eggplant heirlooms are shown in Table 5. Fruit color varied greatly among different genotypes. Regarding the skin Lightness $\left(\mathrm{L}^{*}\right)$, higher values were obtained from genotypes which were mostly, cream, yellowish, orange-yellowish or goldenred in color $\left(\mathrm{L}^{*}>70\right)$. When $\mathrm{L}^{*}$ values were ranged from 48 to 58 together with $h^{\circ}$ values ranged from 80 to 112 , it is observed that these genotypes' skin color were generally greenish-yellow, chartreuse or green in color. Regarding the $\mathrm{b}^{*}$ values, very low values near or below zero mostly indicated that the skin color was cyan (if $h$ ${ }^{\circ}>285$ ); blue (if $h^{\circ}>240$ ); purple (if $h^{\circ}>285$ ); magenta (if $b^{\circ}$ $>350)$. It is observed from the research, that fruits which had lower $b^{\circ}$ values $\left(1.05<b^{\circ}<12.60\right)$ were found to be red in color. Chroma values were varied from 3.82 to 24.26 .

Totally 40 basic morphological descriptors were used for to determine the phylogenetic relationships among the Burdur local heirlooms. The Eigen value was 84. A 2-way Mantel test (Mantel, 1967) method was performed. Approximate Mantel $\mathrm{t}$ - statistic test were $\mathrm{t}=10.0925, \mathrm{p}=1.0000$. The matrix correlation $(\mathrm{r})$ was 0.72 . The similarity rates according to the coefficient similarity of genotypes ranged between 0.29 and 0.91 .

Two major groups were revealed using the dendrogram generated by the UPGMA method using morphological data (Fig. 2). First group (Group A) was consisted of YRL 75, YRL 65, YRL 61 and YRL 59. These genotypes showed low genetic similarity with reference genotypes and cultivar (Long purple). Second group was divided into three subgroups. Group B consist of YRL 19 which showed high genetic similarity with reference cultivar YRL 68 (Long purple). The highest genetic similarity was observed in Group D between genotypes YRL 47 and YRL 51. The fruits of eggplant Burdur heirlooms belonging to the Group C are shown in Fig. 3.

Table 4. Some of the phenotypic descriptors related important variable traits of eggplant

\begin{tabular}{|c|c|c|c|c|c|c|c|c|c|c|c|c|c|c|c|}
\hline Genotype & $\begin{array}{c}\text { Fruit } \\
\text { calyx } \\
\text { prickles }\end{array}$ & $\begin{array}{c}\text { Fruit length } \\
\text { coveredbythe } \\
\text { calyx }\end{array}$ & $\begin{array}{c}\text { Degree of } \\
\text { fruitcarvature }\end{array}$ & $\begin{array}{l}\text { Fruit } \\
\text { grooved }\end{array}$ & $\begin{array}{l}\text { Dominated } \\
\text { fruitcolor }\end{array}$ & $\begin{array}{c}\text { Distribution of } \\
\text { dominant } \\
\text { color }\end{array}$ & $\begin{array}{c}\text { Fruit } \\
\text { flesh } \\
\text { firmness }\end{array}$ & $\begin{array}{l}\text { Fruitflech } \\
\text { color }\end{array}$ & $\begin{array}{l}\text { Seed } \\
\text { content }\end{array}$ & $\begin{array}{c}\text { Seed } \\
\text { maturity }\end{array}$ & $\begin{array}{l}\text { Hole } \\
\text { inthe } \\
\text { fruit }\end{array}$ & Brix & $\begin{array}{c}\text { Fruit } \\
\text { diameter } \\
(\mathrm{mm}) \\
\end{array}$ & $\begin{array}{c}\text { Fruit } \\
\text { lenght } \\
(\mathrm{cm})\end{array}$ & $\begin{array}{c}\text { Fruit } \\
\text { weight } \\
(\mathrm{kg})\end{array}$ \\
\hline YRL 1 & few & between $20-70 \%$ & slight & absent & green & mottled & floppy & greenish-cream & intermediate & mature & absent & 5.2 & 34.80 & 19.8 & 0.116 \\
\hline YRL3 & few & between $20-70 \%$ & slight & present & purple & striped & tightly & white & many & mature & present & 4.2 & 49.79 & 14.2 & 0.140 \\
\hline YRL6 & mid & between $20-70 \%$ & slight & absent & green & mottled & floppy & greenish-cream & intermediate & immature & absent & 4.2 & 36.60 & 23.3 & 0.140 \\
\hline YRL7 & mid & lessthan $20 \%$ & mid- & absent & black & regular & floppy & cream & intermediate & mature & absent & 5.1 & 40.33 & 18.7 & 0.133 \\
\hline YRL8 & mid & lessthan $20 \%$ & slight & present & purple & regular & tightly & greenish-cream & intermediate & mature & present & 47 & 46.86 & 15.7 & 0.133 \\
\hline YRL9 & few & between $20-70 \%$ & slight & absent & black & regular & tightly & greenish-cream & intermediate & mature & absent & 3.8 & 45.38 & 18.5 & 0.138 \\
\hline YRL 12 & few & lessthan $20 \%$ & slight & absent & purple & regular & spongy & white & intermediate & mature & present & 28 & 40.98 & 20.5 & 0.125 \\
\hline YRL 14 & mid & between $20-70 \%$ & none & absent & black & regular & tightly & greenish-cream & few & mature & present & 43 & 43.60 & 17.2 & 0.133 \\
\hline YRL 15 & mid & between $20-70 \%$ & slight & present & black & regular & tightly & greenish-cream & few & immature & present & 6.1 & 35.07 & 18.8 & 0.110 \\
\hline YRL 19 & few & between $20-70 \%$ & slight & absent & black & regular & tightly & greenish-cream & intermediate & mature & absent & 4.1 & 4732 & 175 & 0.146 \\
\hline YRL 20 & mid & between $20-70 \%$ & slight & absent & black & regular & spongy & greenish-cream & many & immature & present & 5.2 & 42.17 & 20.6 & 0.160 \\
\hline YRL 24 & few & lessthan $20 \%$ & slight & absent & purple & regular & spongy & greenish-cream & few & immature & present & 5.2 & 38.96 & 18.3 & 0.100 \\
\hline YRL 26 & few & between $20-70 \%$ & mid- & absent & green & mealy & floppy & white & intermediate & mature & present & 3.2 & 45.73 & 167 & 0.143 \\
\hline YRL 27 & mid & between $20-70 \%$ & slight & absent & purple & mealy & floppy & white & intermediate & mature & present & 4.2 & 58.13 & 18.0 & 0.213 \\
\hline YRL 28 & few & lessthan $20 \%$ & mid- & absent & black & regular & floppy & white & intermediate & mature & present & 3.4 & 53.13 & 16.3 & 0.177 \\
\hline YRL 30 & mid & lessthan $20 \%$ & mid- & absent & purple & mealy & spongy & white & intermediate & immature & present & 3.1 & 47.43 & 14.5 & 0.133 \\
\hline YRL 34 & mid & between $20-70 \%$ & slight & present & purple & mealy & spongy & white & few & mature & present & 3.1 & 54.11 & 22.1 & 0.232 \\
\hline YRL 35 & mid & between $20-70 \%$ & mid- & absent & purple & mealy & spongy & white & few & mature & present & 3.0 & 53.66 & 17.5 & 0.207 \\
\hline YRL 36 & mid & between $20-70 \%$ & mid- & absent & purple & mealy & floppy & white & intermediate & mature & present & 3.2 & 5205 & 14.8 & 0.163 \\
\hline YRL 43 & mid & between $20-70 \%$ & none & present & purple & mottled & spongy & white & intermediate & immature & present & 3.2 & 57.87 & 15.1 & 0.187 \\
\hline YRL 44 & mid & between $20-70 \%$ & slight & absent & purple & mealy & spongy & white & intermediate & mature & present & 3.4 & 51.29 & 15.2 & 0.168 \\
\hline YRL 45 & mid & between $20-70 \%$ & slight & present & purple & mealy & tightly & white & few & mature & present & 39 & 50.50 & 14.6 & 0.173 \\
\hline YRL 46 & mid & lessthan $20 \%$ & mid- & present & purple & regular & floppy & white & intermediate & mature & absent & 4.1 & 4279 & 16.4 & 0.125 \\
\hline YRL47 & few & lessthan $20 \%$ & mid- & present & purple & regular & floppy & white & intermediate & immature & absent & 4.1 & 35.05 & 18.0 & 0.127 \\
\hline YRL 49 & few & less than $20 \%$ & mid- & absent & purple & regular & tightly & white & intermediate & immature & present & 3.8 & 44.98 & 19.7 & 0.153 \\
\hline YRL 50 & mid & lessthan 20\% & mid- & absent & purple & regular & tightly & white & intermediate & mature & present & 3.1 & 43.15 & 19.2 & 0.143 \\
\hline YRL 51 & few & lessthan $20 \%$ & mid- & present & purple & regular & floppy & white & intermediate & mature & absent & 3.1 & 44.82 & 17.4 & 0.147 \\
\hline YRL 52 & few & lessthan 20\% & mid- & absent & purple & regular & tightly & white & intermediate & mature & present & 2.9 & 42.73 & 175 & 0.133 \\
\hline YRL 57 & few & between $20-70 \%$ & mid- & absent & green & mottled & tightly & greenish-cream & intermediate & immature & present & 5.1 & 32.67 & 19.1 & 0.100 \\
\hline YRL 58 & few & lessthan $20 \%$ & mid- & absent & purple & regular & tightly & white & few & mature & present & 4.2 & 47.03 & 18.5 & 0.148 \\
\hline YRL59 & mid & between $20-70 \%$ & mid- & absent & purple & mottled & floppy & white & intermediate & mature & present & 47 & 51.40 & 13.0 & 0.155 \\
\hline YRL61 & mid & between $20-70 \%$ & none & present & purple & regular & tightly & white & intermediate & mature & present & 4.1 & 64.76 & 10.9 & 0.183 \\
\hline YRL 62 & few & between $20-70 \%$ & slight & absent & black & regular & tightly & greenish-cream & few & immature & present & 4.9 & 46.09 & 16.3 & 0.143 \\
\hline YRL 64 & few & lessthan $20 \%$ & slight & absent & purple & regular & floppy & white & intermediate & mature & present & 3.8 & 51.86 & 22.9 & 0.228 \\
\hline YRL 65 & mid & lessthan $20 \%$ & slight & present & purple & mottled & tightly & white & intermediate & mature & present & 3.8 & 73.22 & 11.6 & 0.235 \\
\hline YRL 68 & mid & between $20-70 \%$ & slight & present & black & regular & tightly & greenish-cream & medium & immature & present & 3.1 & 50.76 & 14.2 & 0.150 \\
\hline YRL75 & mid & between $20-70 \%$ & none & present & black & regular & tightly & greenish-cream & intermediate & mature & present & 4.2 & 65.97 & 11.9 & 0.137 \\
\hline YRL79 & few & between $20-70 \%$ & slight & present & green & mottled & floppy & greenish-cream & intermediate & immature & present & 4.2 & 47.71 & 13.6 & 0.122 \\
\hline
\end{tabular}


104

Table 5. Skin color characteristics of eggplant

\begin{tabular}{|c|c|c|c|c|c|c|}
\hline Genotype & $\mathrm{L}^{*}$ & $a^{*}$ & $\mathrm{~b}^{*}$ & $C$ & $b^{\circ}$ & color \\
\hline YRL 1 & $48.33 \pm 6.38$ & $-0.67 \pm 4.80$ & $18.38 \pm 4.04$ & $19.04 \pm 3.88$ & $88.77 \pm 15.99^{\mathrm{X}}$ & chartreuse \\
\hline YRL 3 & $73.36 \pm 4.06$ & $9.05 \pm 2.52$ & $6.31 \pm 1.09$ & $11.27 \pm 1.52$ & $36.39 \pm 11.67$ & yellowish-orange \\
\hline YRL 6 & $51.54 \pm 8.50$ & $-6.18 \pm 5.43$ & $22.47 \pm 6.03$ & $23.75 \pm 6.71$ & $101.46 \pm 13.81$ & chartreuse \\
\hline YRL 7 & $26.21 \pm 0.66$ & $6.03 \pm 1.76$ & $0.36 \pm 0.51$ & $6.05 \pm 1.78$ & $62.44 \pm 131.52$ & yellowish \\
\hline YRL 8 & $31.83 \pm 3.32$ & $11.44 \pm 2.19$ & $2.69 \pm 1.07$ & $11.78 \pm 2.31$ & $12.60 \pm 4.48$ & redish \\
\hline YRL 9 & $29.28 \pm 3.64$ & $10.22 \pm 3.40$ & $1.25 \pm 1.45$ & $10.38 \pm 3.48$ & $5.85 \pm 6.76$ & redish \\
\hline YRL 12 & $31.71 \pm 3.20$ & $21.64 \pm 2.52$ & $-0.98 \pm 0.75$ & $21.67 \pm 2.54$ & $357.56 \pm 1.74$ & magenta \\
\hline YRL 14 & $25.83 \pm 0.99$ & $4.97 \pm 0.79$ & $0.36 \pm 0.88$ & $5.03 \pm 0.93$ & $182.80 \pm 175.41$ & cyan \\
\hline YRL 15 & $25.23 \pm 0.22$ & $4.07 \pm 1.31$ & $0.002 \pm 0.26$ & $4.08 \pm 1.31$ & $299.07 \pm 131.60$ & purple \\
\hline YRL 19 & $25.48 \pm 0.51$ & $3.81 \pm 0.78$ & $-0.03 \pm 0.22$ & $3.82 \pm 0.79$ & $238.99 \pm 167.40$ & blue \\
\hline YRL 20 & $25.82 \pm 0.92$ & $4.84 \pm 1.15$ & $0.003 \pm 0.28$ & $4.85 \pm 1.15$ & $299.43 \pm 131.95$ & purple \\
\hline YRL 24 & $29.02 \pm 3.24$ & $17.85 \pm 4.63$ & $-0.42 \pm 0.54$ & $17.86 \pm 4.65$ & $243.94 \pm 146.42$ & blue \\
\hline YRL 26 & $55.22 \pm 5.86$ & $-7.43 \pm 2.45$ & $17.56 \pm 3.60$ & $19.10 \pm 4.20$ & $112.31 \pm 3.60$ & greenish \\
\hline YRL 27 & $68.54 \pm 5.60$ & $8.83 \pm 2.37$ & $5.71 \pm 1.76$ & $10.88 \pm 1.00$ & $34.29 \pm 14.89$ & orange \\
\hline YRL 28 & $29.89 \pm 2.86$ & $9.70 \pm 0.84$ & $1.57 \pm 1.36$ & $9.93 \pm 0.72$ & $9.29 \pm 8.33$ & red \\
\hline YRL 30 & $66.80 \pm 2.88$ & $11.23 \pm 1.66$ & $6.30 \pm 1.17$ & $12.99 \pm 1.04$ & $29.80 \pm 7.71$ & orange \\
\hline YRL 34 & $77.73 \pm 3.23$ & $4.64 \pm 1.69$ & $9.76 \pm 1.00$ & $10.98 \pm 0.16$ & $64.53 \pm 10.30$ & cream-yellowish \\
\hline YRL 35 & $75.07 \pm 2.53$ & $6.01 \pm 1.29$ & $8.28 \pm 1.20$ & $10.38 \pm 0.33$ & $53.91 \pm 9.74$ & yellow \\
\hline YRL 36 & $72.34 \pm 4.46$ & $8.41 \pm 2.74$ & $6.96 \pm 1.55$ & $11.30 \pm 1.24$ & $41.16 \pm 14.66$ & orange-yellow \\
\hline YRL 43 & $56.55 \pm 9.09$ & $19.35 \pm 5.14$ & $2.09 \pm 2.76$ & $19.81 \pm 4.51$ & $68.93 \pm 128.77$ & yellow \\
\hline YRL 44 & $72.50 \pm 8.32$ & $7.51 \pm 4.42$ & $7.49 \pm 2.90$ & $11.58 \pm 2.54$ & $48.10 \pm 21.20$ & goldenrod \\
\hline YRL 45 & $76.29 \pm 4.16$ & $5.00 \pm 2.26$ & $10.97 \pm 2.10$ & $12.40 \pm 1.05$ & $64.49 \pm 13.53$ & yellow \\
\hline YRL 46 & $37.41 \pm 5.48$ & $22.90 \pm 1.68$ & $-0.49 \pm 1.07$ & $22.94 \pm 1.68$ & $298.96 \pm 131.62$ & purple \\
\hline YRL 47 & $32.80 \pm 4.44$ & $20.59 \pm 3.19$ & $-0.50 \pm 1.36$ & $20.64 \pm 3.23$ & $357.03 \pm 2.04$ & magenta \\
\hline YRL 49 & $32.04 \pm 3.54$ & $23.02 \pm 2.99$ & $-0.51 \pm 0.47$ & $23.04 \pm 3.00$ & $240.48 \pm 166.70$ & blue \\
\hline YRL 50 & $36.77 \pm 6.11$ & $22.17 \pm 2.18$ & $-0.07 \pm 1.29$ & $22.20 \pm 2.15$ & $179.90 \pm 177.00$ & cyan \\
\hline YRL 51 & $37.55 \pm 4.73$ & $21.85 \pm 3.29$ & $-0.89 \pm 0.93$ & $21.88 \pm 3.30$ & $285.88 \pm 142.39$ & purple \\
\hline YRL 52 & $35.51 \pm 2.61$ & $23.76 \pm 1.51$ & $-1.37 \pm 0.48$ & $23.81 \pm 1.49$ & $356.67 \pm 1.24$ & magenta \\
\hline YRL 57 & $44.44 \pm 8.19$ & $4.04 \pm 6.62$ & $9.70 \pm 5.12$ & $13.35 \pm 1.43$ & $63.87 \pm 38.34$ & yellowish \\
\hline YRL 58 & $37.80 \pm 5.11$ & $23.66 \pm 2.46$ & $-1.61 \pm 0.66$ & $23.72 \pm 2.43$ & $356.05 \pm 1.90$ & magenta \\
\hline YRL 59 & $56.43 \pm 6.66$ & $18.22 \pm 4.99$ & $1.86 \pm 2.45$ & $18.55 \pm 4.72$ & $80.11 \pm 137.94$ & greenish-yellow \\
\hline YRL 61 & $51.83 \pm 4.36$ & $14.86 \pm 2.14$ & $0.41 \pm 3.95$ & $15.45 \pm 1.56$ & $242.80 \pm 156.44$ & blue \\
\hline YRL 62 & $31.03 \pm 5.72$ & $10.50 \pm 2.59$ & $2.53 \pm 2.32$ & $10.96 \pm 2.90$ & $12.19 \pm 9.68$ & redish \\
\hline YRL 64 & $37.51 \pm 4.79$ & $24.23 \pm 0.59$ & $0.02 \pm 1.29$ & $24.26 \pm 0.61$ & $285.99 \pm 142.37$ & purple \\
\hline YRL 65 & $73.19 \pm 4.48$ & $8.17 \pm 2.89$ & $7.90 \pm 0.91$ & $11.66 \pm 1.61$ & $45.71 \pm 12.66$ & orange-yellow \\
\hline YRL 68 & $35.81 \pm 5.99$ & $9.95 \pm 2.41$ & $6.17 \pm 3.81$ & $12.01 \pm 3.63$ & $27.50 \pm 15.13$ & dark-orange \\
\hline YRL 75 & $25.39 \pm 0.72$ & $6.03 \pm 1.01$ & $0.12 \pm 0.14$ & $6.03 \pm 1.01$ & $1.05 \pm 1.04$ & redish \\
\hline YRL 79 & $57.34 \pm 3.69$ & $0.12 \pm 2.26$ & $19.50 \pm 2.14$ & $19.64 \pm 2.08$ & $88.98 \pm 6.91$ & chartreuse \\
\hline
\end{tabular}

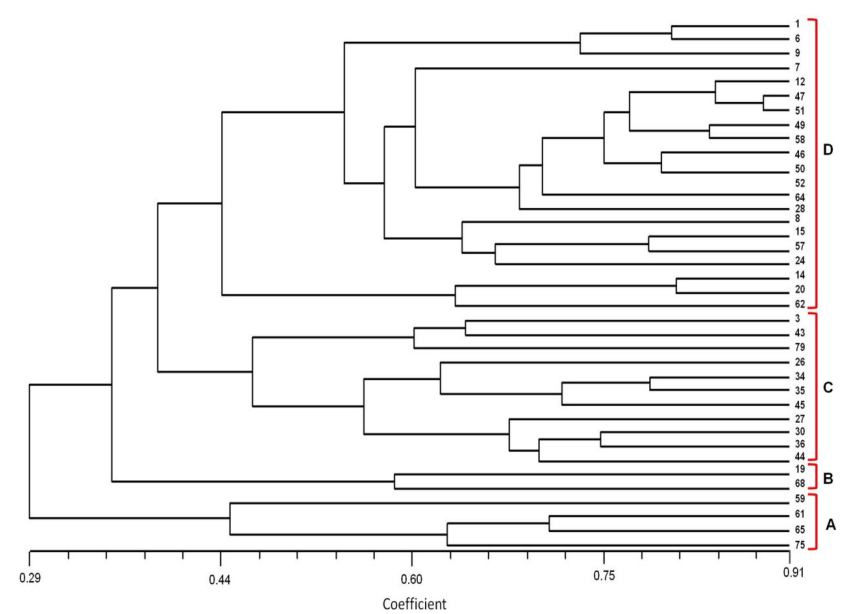

Fig. 2. UPGMA dendrogram showing phylogenetic relationships of local Burdur eggplant heirlooms together with reference cultivars using morphological data

A total of 65 amplified RAPD bands were generated. Twenty nine bands were polymorphic and the mean percentage of polymorphism was $44.61 \%$. OPO-10 primer produced the maximum numbers of bands (18). Although the OPB-07 primer produced the minimal number of bands (8), it revealed a $100 \%$ polymorphism (Table 6). The OPH-2, OPB-07, OPO10 and OPL-16 primers' PCR products and their band patterns are shown in Fig. 4a-d.
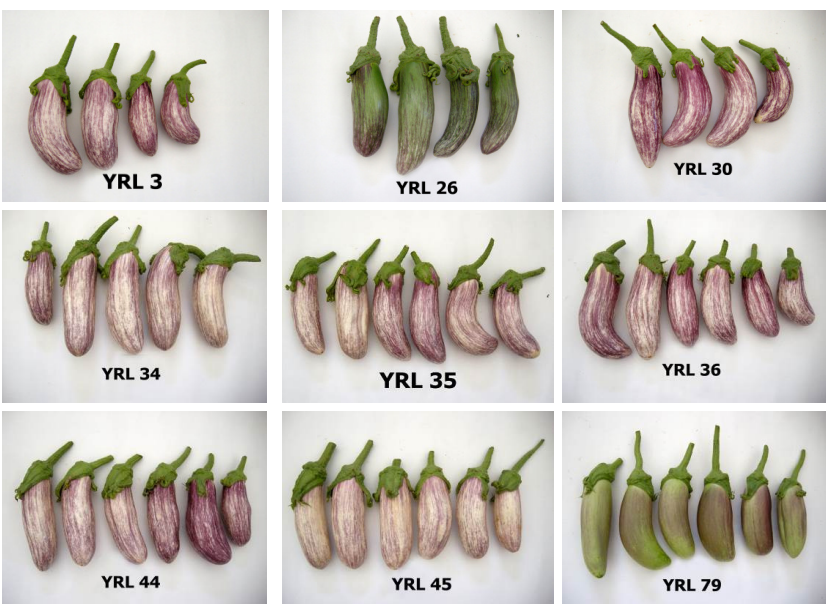

Fig. 3. The fruits of Burdur eggplant heirlooms belonging to the Group C

Table 6. Primer code, sequence, GC content, number of polymorphic bands and percent polymorphism of each RAPD primer used

\begin{tabular}{lcccc}
\hline $\begin{array}{l}\text { Primer } \\
\text { code }\end{array}$ & Nucleotide sequence & $\begin{array}{c}\text { No. of } \\
\text { amplified } \\
\text { bands }\end{array}$ & $\begin{array}{c}\text { No. of } \\
\text { polymorphic } \\
\text { bands }\end{array}$ & $\begin{array}{c}\text { Polymorphic } \\
\text { bands (\%) }\end{array}$ \\
\hline OPH-02 & 5'-TCG GAC GTG A-3' & 14 & 7 & 50 \\
OPL-04 & 5'-GAC TGC ACA C-3' & 16 & 4 & 25 \\
\hline OPB-07 & 5'-GGT GAC GCA G-3' & 8 & 8 & 100 \\
OPO-10 & 5'-TCA GAG CGC C-3' & 18 & 5 & 27.77 \\
OPL-16 & 5'-AGG TTG CAG G-3' & 9 & 5 & 55.55 \\
TOTAL & & $\mathbf{6 5}$ & $\mathbf{2 9}$ & $\mathbf{4 4 . 6 1}$ \\
\hline
\end{tabular}


The phylogenetic relationships among 38 genotypes were evaluated using 29 polymorphic loci of the genomic DNA generated using randomly amplified polymorphic DNA (RAPD) technique. The approximate Mantel t-test statistic were $\mathrm{t}=8.9263, \mathrm{p}=1.0000$. The matrix correlation $(\mathrm{r})$ was found to be 0.64 . The similarity rates according to the coefficient similarity of genotypes ranged between 0.84 and 0.98 .

Using molecular data two major groups emerged in the dendrogram generated with UPGMA method (Fig. 5). First group branched into two subgroups and were showed in brackets as Group A and Group B. Group A was consisted of YRL 15 and YRL 27. Group E included most of the genotypes had high similarities with reference cultivar. The highest genetic similarity was observed in Group D with 98\% percentage similarity index between genotypes YRL 6 and YRL 44. YRL 1 and YRL 27 were detected as the most distantly genotypes from each other in the cluster. Fruits of Burdur eggplant heirlooms YRL 6 and YRL 44, which showed highest genetic similarity with $98 \%$ percentage are shown in Fig. 6. Also, the fruits of
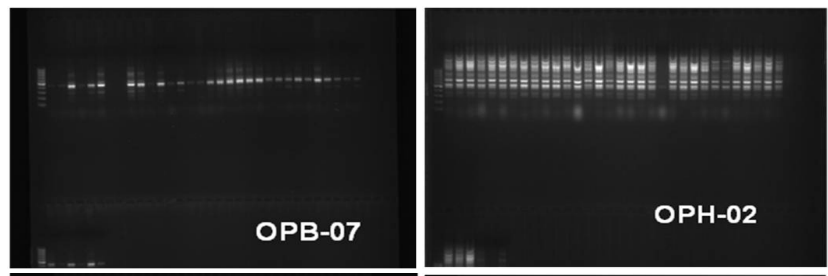

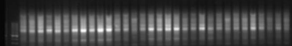
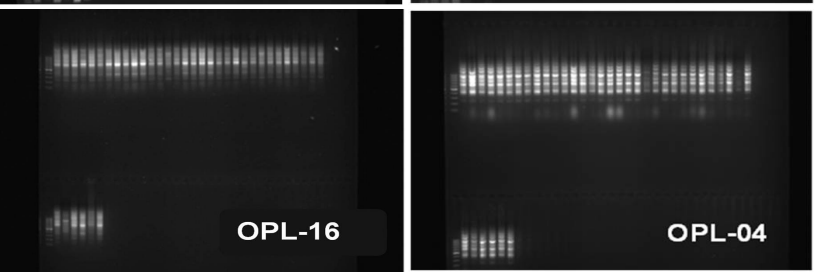

Fig. 4. PCR products of Burdur eggplant heirlooms generated using RAPD primers a:OPB-07, b:OPH-02, c:OPO-016, d:OPL-04

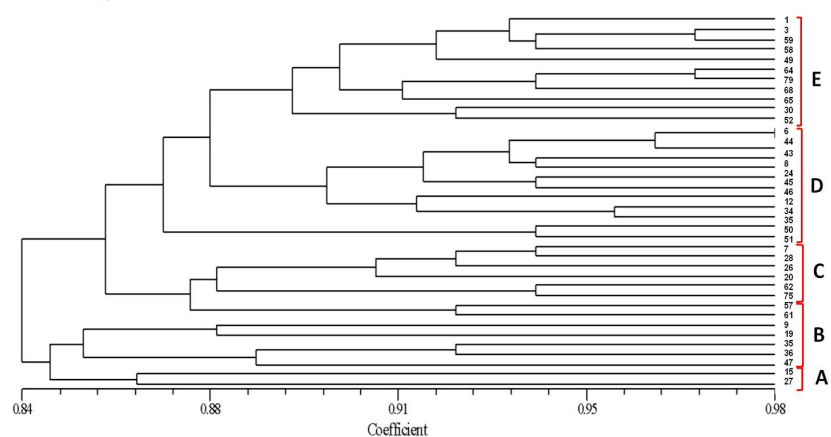

Fig. 5. UPGMA dendrogram showing phylogenetic relationships of local Burdur eggplant heirlooms together with reference cultivars using molecular data

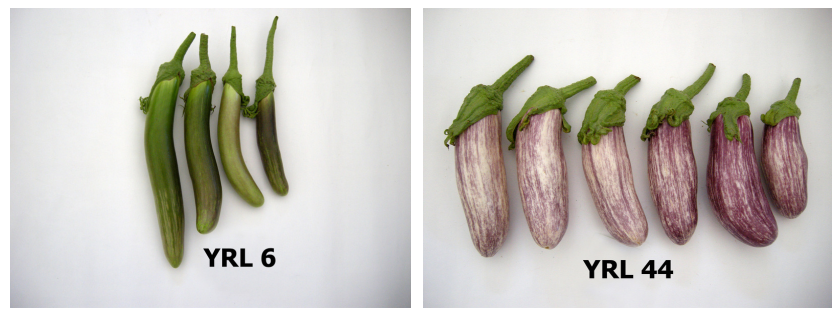

Fig. 6. Fruits of Burdur eggplant heirlooms YRL 6 and YRL 44, which showed highest genetic similarity with $98 \%$ percentage
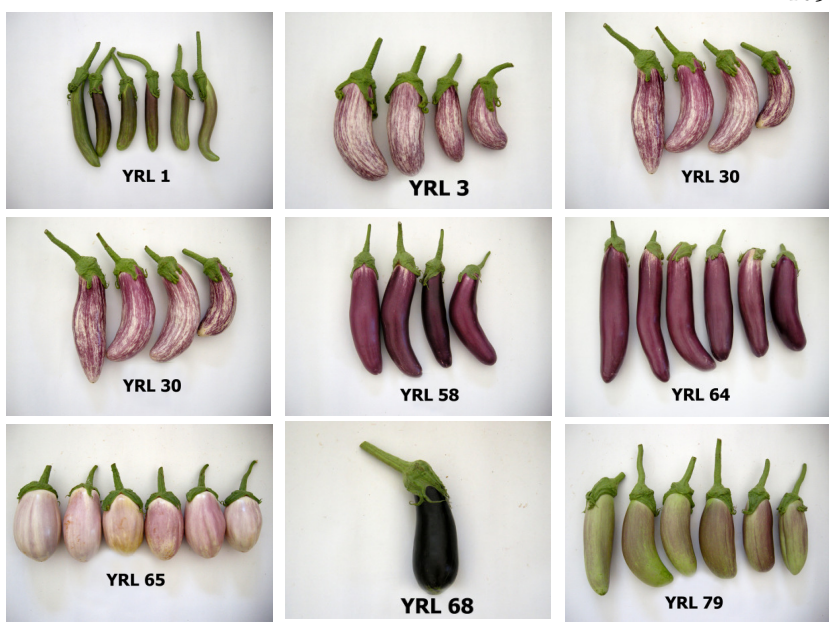

Fig. 7. Fruits of eggplant Burdur heirlooms with reference cultivar (YRL 68) situated in GROUP E generated by UPGMA using molecular data

Burdur heirlooms which grouped together in cluster $\mathrm{E}$ in the UPGMA dendrogram using molecular data and reference culture are shown in Fig. 7.

\section{Discussions}

Eggplant has a wide genetic diversity in the regions where it is cultivated, although they are not native to the region (Muñoz-Falcón et al., 2008). In spite of the fact that Turkey is not the place origin of eggplant, wide genetic diversity has been reported in Turkey (Demir $e t$ al., 2010; Tümbilen $e t$ al., 2011a, 2011b). It is clearly evident in this study data that Burdur, which is a small geographical region, had a rich genetic diversity. Also, a wide genetic variability was determined in both Spain and Jordan local genotypes (Prohens et al., 2003). Local genotypes can contribute to enhancing the gene pool used in breeding studies and to help increase heterosis (Muñoz-Falcón et al., 2009). In recent years, some factors like cultivation of commercial varieties instead of heirlooms, construction of buildings on agricultural land, and innovation in cultivation methods have led to erosion of plant genetic resources (Cericola et al., 2013). Therefore, there is a need collecting and identification of local heirlooms before they disappear (Muñoz-Falcón et al., 2008).

Some characters that contributed to genetic diversity were as flowering dates, the number of seeds per fruits, fruit features, and the growth pattern of plants. These features are controlled by several genes in eggplant (Frary and Doğanlar, 2003). Solanum melongena accessions could characterize these descriptors like bigger and flabby fruits, less flowers/inflorescence, few fruits/plant and higher acidity etc. compared to the wild relatives (Polignano et al., 2010). Consistent with previously works, a higher diversity for most morphological descriptors was recorded in the collection of Burdur local heirlooms identified in this study. Fruit color can be cream, green, red, reddish-purple, dark purple or black, and some varieties produce fruit which is where the genetic variation necessary for future varietal improvement and for addressing future breeding challenges will be found.

Molecular markers linked with agronomic traits are useful tools for marker assisted selection and mapping candidate genes studies in breeding programs (Nunome et al., 2009; 
106

Wang et al., 2010). Some of the RAPD markers used were determined to have relationship with coloring of stem, calyx and fruiting in eggplant (Frary et al., 2003). This provides a great advantage for identifying features affecting by ecological conditions (Biswas et al., 2009; Nunome et al., 2001). Relationships among eggplant materials have been studied by molecular studies for use by eggplant breeders (Furini and Wunder, 2004). As mentioned above the RAPD markers revealed as a potential useful tool for determination of genetic diversity. It was found that RAPD analysis in eggplant with four primers had been adequate to identify of genetic diversity (Biswas et al., 2009). Also, RAPD markers were more effective than ISSR for revealing genetic diversification as reported by Ali et al. (2011). Tiwari et al. (2009) previously reported that even two of the 29 RAPD primers were found to be sufficient for identification of local cultivars. All RAPD markers used in the study have produced polymorphic bands as expected. However, if fruit criteria are taken into consideration for distinguishing in the dendrogram and grouping, it is not enough for the breeders. More informative DNA markers can be used to provide better progress in eggplant breeding studies (Stàgel et al., 2008). Simple Sequence Repeats (SSRs) methods were found more successful for distinguishing closely related eggplant cultivars (Hurtado et al., 2012; Muñoz-Falcón et al., 2009; Prohens et al., 2008). The approach of using SSR markers instead of RAPD markers can provide better results in the phylogenetic relationships studies. Muñoz-Falcón et al. (2009) reported that if the morphological and molecular data are considered together, they can be provide sufficient and useful information for the breeders. Similarly to the findings of other studies, we suggest that the molecular evidences need to be supplemented by morphological data to validate the phylogenetic relationships among the genotypes. It is very important to note that the genetic variations can't be detected by only showing a dendrograms generated by molecular data.

\section{Conclusions}

The local populations are of great importance for the breeders so that they adapted well to their cultivated areas. There is a need collecting and identification of these heirlooms before integrated to the breeding programs. The aim of the study was to investigate the diversity among heirlooms cultivated in Burdur province. A high genetic diversity was determined widely among them. These materials can be of a potential value for the breeders.

\section{Acknowledgements}

This research was financially supported by grants from the General Directorate of Agricultural Research and Policy, Republic of Turkey Ministry of Food, Agriculture and Livestock under BBSS-10-12 project number.

\section{References}

Ali Z, Xu ZL, Zhang D Y, He XL, Bahadur S, Yi JX (2011). Molecular diversity analysis of eggplant (Solanum melongena) genetic resources. Genetics and Molecular Research 10(2):11411155.
Barchi L, Lanteri S, Portis E, Stàgel A, Valè G, Toppino L, Rotino GL (2010). Segregation distortion and linkage analysis in eggplant (Solanum melongena L.). Genome 53(10):805-815.

Biswas MS, Akhond MAY, Al-Amin A, Khatun M, Kabir MR (2009). Genetic relationship among ten promising eggplant varieties using RAPD markers. Plant Tissue Culture and Biotechnology 19(2):119-126.

Cericola F, Portis E, Toppino L, Barchi L, Acciarri N, Ciriaci T, Sala T, Rotino GL, Lanteri S (2013). The population structure and diversity of eggplant from Asia and the Mediterranean basin. PloS ONE 8(9):DOI:10.1371/journal.pone.0073702.

Daunay MC, Lester NR, Gebhardt C, Hennart W, Jahn M (2001). Genetic resources of eggplant (Solanum melongena L.) and allied species: a new challenge for molecular genetics and eggplant breeders. In: Van Den Berg RG, Barendse GW, Mariani C (Eds). Solanaceae V. Nijmegen University, Press Nijgemen, The Netherlands, pp. 251-274.

Daunay MC, Janick J (2007). History and Iconography of Eggplant. Chronica Horticulturae 47(3):16-22.

Demir K, Bakır M, Sarıkamış G, Acunalp S (2010). Genetic diversity of eggplant (Solanum melongena) germplasm from Turkey assessed by SSR and RAPD markers. Genetics and Molecular Research 9(3):1568-1576.

Ekiz H, Boyacı HF (2001). Pepper and eggplant varieties in greenhouses on the coast of Mediterranean in Antalya. XI ${ }^{\text {th }}$ Meeting on Genetics and Breeding of Capsicum and Eggplant, 9-12 April, Antalya, 241245.

Frary A, Doganlar S (2003). Comparative genetics of crop plant domestication and evolution. Turkish Journal of Agriculture and Forestry 27:59-69.

Frary A, Doganlar S, Daunay MC, Tanksley SD (2003). QTL analysis of morphological traits in eggplant and implications for conservation of gene function during evolution of Solanaceous species. Theoretical and Applied Genetics 107(2):359-370.

Frary A, Doganlar S, Daunay MC (2007). Eggplant, p. 287-314. In: Genome Mapping and Molecular Breeding in Plants. Kole C (Ed). Springer, Acad Press.

Fukuoka H, Yamaguchi H, Nunome T, Negoro S, Miyatake K, Ohyama A (2010). Accumulation, functional annotation, and comparative analysis of expressed sequence tags in eggplant (Solanum melongena L.), the third pole of the genus Solanum species after tomato and potato. Gene 450(1-2):76-84.

Furini A, Wunder J (2004). Analysis of eggplant (Solanum melongena L.) related germplasm: morphological and AFLP data contribute to phylogenetic interpretations and germplasm utilization. Theoretical and Applied Genetics 108(2):197-208.

Hurtado M, Vilanova S, Plazas M, Gramazio P, Fonseka HH, Fonseka R, Prohens J (2012). Diversity and relationships of eggplants from three geographically distant secondary centers of diversity. PLoS ONE 7(7):DOI:10.1371/journal.pone.0041748.

Janick J (2001). Asian crops in North America. HortTechnology 11:510-513.

Kashyap V, Kumar SV, Collonnier C, Fusari F, Haicour R, Rotino 
GL, Sihachakr D, Rajam RM (2003). Biotechnology of eggplant. Scientia Horticulturae 97:1-25.

Kowalska G (2008). Flowering biology of eggplant and procedures intensifying fruit-set. Acta Scientiarum Polonorum, Hortorum Cultus 7(4):63-76.

Li H, Chen H, Zhuang T, Chen J (2010). Analysis of genetic variation in eggplant and related Solanum species using sequencerelated amplified polymorphism markers. Scientia Horticulturae 125(1):19-24.

Mantel NA (1967). The detection of disease clustering and a generalized regression approach. Cancer research 27(2 Part 1):209-220.

Muñoz-Falcón JE, Prohens J, Vilanova S, Nuez F (2008). Characterization, diversity, and relationships of the Spanish striped (Listada) eggplants: a model for the enhancement and protection of local heirlooms. Euphytica 164(2):405-419.

Muñoz-Falcón JE, Prohens J, Vilanova S, Ribas F, Castro A, Nuez F (2009). Distinguishing a protected geographical indication vegetable (Almagro eggplant) from closely related varieties with selected morphological traits and molecular markers. Journal of the Science of Food and Agriculture 89(2):320-328.

Muñoz-Falcón JE, Prohens J, Vilanova S, Nuez F (2009). Diversity in commercial varieties and landraces of black eggplants and implications for broadening the breeders' gene pool. Annals of Applied Biology 154(3):453-465.

Mutlu N, Boyaci FH, Göçmen M, Abak K (2008). Development of SRAP, SRAP-RGA, RAPD and SCAR markers linked with a Fusarium wilt resistance gene in eggplant. Theoretical and Applied Genetics 117(8):1303-1312.

Nunome $T$, Ishiguro $K$, Yoshida $T$, Hirai $M$ (2001). Mapping of fruit shape and color development traits in eggplant (Solanum melongena L.) based on RAPD and AFLP markers. Breeding Science 51(1):19-26.

Nunome T, Negoro S, Kono I, Kanamori H, Miyatake K, Yamaguchi H, Ohyama A, Fukuoka H (2009). Development of SSR markers derived from SSR-enriched genomic library of eggplant (Solanum melongena L.). Theoretical and Applied Genetics 119(6):11431153.

Polignano G, Uggenti P, Bisignano V, Della Gatta C (2010). Genetic divergence analysis in eggplant (Solanum melongena $\mathrm{L}$.) and allied species. Genetic Resources and Crop Evolution 57(2):171-181.

Prohens J, Valcarcel JV, Fernandez de Cordova P, Nuez F (2003). Characterization and typification on Spanish eggplant landraces. Capsicum Eggplant News 22:135-138.
Prohens J, Muñoz-Falcón JE, Vilanova S, Nuez F (2008). Use of molecular markers for the enhancement of local varieties of vegetables for Protected Designations of Origin and Geographical Indications. Bulletin of University of Agricultural Sciences and Veterinary Medicine Cluj-Napoca. Horticulture 65(1):16-20.

Prohens J, Muñoz-Falcón JE, Vilanova S, Nuez F (2011). Comparison of Morphological, AFLP and SSR Markers for the Protection of Eggplant Germplasm. Acta Hort 898:123-131.

Reed DH, Frankham R (2001). How closely correlated are molecular and quantitative measures of genetic variation? a metaanalysis. Evolution 55(6):1095-1103.

Rohlf FJ (1998). NTSYS-PC: Numerical taxonomy and multivariate analysis system. Release 2.20j. Exeter Software, Setauket, N.Y.

Sekara A, Cebula S, Kunicki E (2007). Cultivated eggplants - origin, breeding objectives and genetic resources, a review. Folia Horticulturae 19(1):97-114.

Stàgel A, Portis E, Toppino L, Rotino GL, Lanteri S (2008). Genebased microsatellite development for mapping and phylogeny studies in eggplant. BMC Genomics 9(1):357.

Swarup V (1995, March). Genetic resources and breeding of aubergine (Solanum melongena L.). In I International Symposium on Solanacea for Fresh Market 412 pp. 71-79.

Tiwari SK, Karihaloo JL, Nowsheen H, Gaikwad AB (2009). Molecular characterization of brinjal (Solanum melongena L.) cultivars using RAPD and ISSR markers. Journal of Plant Biochemistry and Biotechnology 18(2):189-195.

Tümbilen Y, Frary A, Daunay MC, Doganlar S (2011). Application of EST-SSRs to examine genetic diversity in eggplant and its close relatives. Turkish Journal of Biology 35(2):125-136.

Tümbilen Y, Frary A, Mutlu S, Doganlar S (2011). Genetic diversity in Turkish eggplant (Solanum melongena) varieties as determined by morphological and molecular analyses. International Research Journal of Biotechnology 2:16-25.

Wang Q,Zhao F, Sun Q, Yang A (2010). Genetic diversity of eggplant revealed by SSR markers. $4^{\text {th }}$ International Conference on Bioinformatics and Biomedical Engineering June 18-20, 2010 Chengdu, China.

Weese TL, Bohs L (2010). Eggplant origins: out of Africa, into the Orient. Taxon 59(1):49-56. 